NOVA

University of Newcastle Research Online

nova.newcastle.edu.au

Kouretzis, George P.; Sloan, Scott W.; Carter, John P. "Effect of interface friction on tunnel liner internal forces due to seismic S- and P-wave propagation" Soil Dynamics and Earthquake Engineering Vol. 46, p. 41-51 (2013)

Available from: http://dx.doi.org/10.1016/j.soildyn.2012.12.010

Accessed from: http://hdl.handle.net/1959.13/1056700 


\title{
Effect of interface friction on tunnel liner internal forces due to seismic S- and $P$ - wave propagation
}

\author{
George P. Kouretzis ${ }^{1}$, Scott W. Sloan and John P. Carter \\ ARC Centre of Excellence for Geotechnical Science and Engineering, Faculty of Engineering and Built \\ Environment, The University of Newcastle, Callaghan, NSW 2308, Australia
}

The effects on the hoop force and bending moment developed in the lining of a circular tunnel of the contact properties of the soil-lining interface are investigated numerically for both cases of S- and P- seismic wave propagation. Development of a robust model for the dynamic simulation of this problem includes: (i) the implementation of a hysteretic model of the non-linear soil response under cyclic loads in the finite element code ABAQUS; and (ii) validation of the analyses results against centrifuge tests from the literature and closed-from elasticity solutions. Accordingly, a parametric study is conducted to quantify the effect of adopting different values of the friction coefficient of the tunnel liner interface, while assuming that the relaxation load is transferred only to the temporary support shell of the tunnel; a hypothesis applicable mainly to tunnels constructed with the NATM method where an unreinforced concrete final lining is usually installed. Practical findings of this study suggest that the full-slip assumption should be used in conjunction with closed-form expressions for preliminary estimates of the tunnel response. On the contrary, for tunnels where the lining is designed to bear the soil loads, numerical tools should be used for the rational assessment of their seismic response. In the latter case, more experimental studies are needed to evaluate the friction properties at the interface, since common expressions correlating the friction coefficient with the friction angle of the surrounding soil do not appear compatible with the centrifuge test results examined herein.

Keywords: earthquake; tunnel; centrifuge test; interface friction; unreinforced final lining

\footnotetext{
${ }^{1}$ Corresponding author - email: Georgios.Kouretzis@newcastle.edu.au tel. +61 24921 6449, postal address: EA Building, University of Newcastle, Callaghan NSW 2308, Australia
} 


\section{Introduction}

Current state-of-practice in the seismic design of tunnels uses either closed-form linear elastic expressions for preliminary estimates of the internal forces developing in the lining [e.g. 1, 2, 3, 4], or comprehensive numerical analyses of the tunnel lining-soil system [5]. Recent research efforts also include the predictions from centrifuge tests $[6,7,8,9]$. These enrich our understanding of tunnel response under seismic wave propagation and also serve as a valuable tool for assessing the validity of various analysis methodologies and their assumptions.

One of the key issues regarding the simulation of tunnel response is the prediction of the contact behavior between the tunnel lining and surrounding medium. Many analytical solutions from linear elasticity assume either zero friction (the full-slip condition) or perfect bond between the tunnel and its surrounding soil (the no-slip condition), and hence cannot simulate the interface response under cyclic loads reliably. Hashash et al. [10], referring to the solution for shear or S-waves proposed by Wang [1], suggested that for most tunnels the interface condition is actually somewhere between full-slip and no-slip. This implies that both cases should be modeled to calculate the most critical internal forces induced by seismic wave propagation, since the solution for the full-slip condition results in significantly lower hoop forces in the tunnel lining. Indeed, the hoop forces calculated under the no-slip condition may be up to 1,000 times larger than those corresponding to the full-slip assumption. Sedarat et al. [5] investigated the effect of the contact properties using extensive elastic-quasi static numerical analyses which simulated S-wave propagation. Their results underlined the influence of the interface properties on the internal forces developing in the tunnel liner, and they concluded that, since closedform solutions fail to adequately predict the tunnel response, numerical methods should be used instead. 
Interestingly, the state-of-practice in tunnel lining design with numerical methods, especially for tunnels constructed with the NATM method, is generally based on the rather crude assumption of zero friction at the soil-tunnel final lining interface. This is justified by the fact that a smooth waterproofing membrane is usually installed between the tunnel temporary support shell and the final tunnel lining.

Following a brief overview of closed-form solutions for the estimation of tunnel liner forces due to seismic S- and P-wave propagation, this paper describes a numerical model for investigating the effect of friction at the soil-tunnel interface via non-linear dynamic finite element analysis. In order to simulate hysteretic soil response under cyclic loading, a user-defined model subroutine was developed for the finite element code ABAQUS/Standard [11]. The numerical results from this model are compared against the centrifuge test measurements originally presented by Lanzano et al. [6] and Lanzano [9]. This comparison shows the effect of the contact surface behaviour and permits the model to be calibrated and refined.

Accordingly, a simpler variant of the abovementioned model is used for comparison with the closed-form elasticity solutions, and a series of parametric analyses is performed to quantify the effect of interface friction on the results. In contrast to the parametric study of Sedarat et al. [5], the current work examines the effect of compression P-waves as well as S-waves, and the tunnel final lining is assumed not to bear any gravity loads resulting from soil mass relaxation. Thus all loads are assumed to be borne by the temporary tunnel shell created during tunnel excavation, and the final tunnel lining is installed only after the soil mass has reached stress equilibrium, as is normal practice with the NATM method. This assumption applies chiefly to unreinforced concrete final lining sections, which are prone to earthquake effects (compared to standard reinforced concrete), and is based on the following rationale: 
a) The load from the surrounding soil mass acts beneficially under seismic loading, as it induces a pre-stressing compressive axial force, which reduces the eccentricity of the hoop force. However, the question of whether these loads from the temporary excavation will have fully transferred to the final lining at the time of an earthquake event is uncertain.

b) Unreinforced concrete tunnel sections are constructed in competent ground conditions, where long-term loads on the final lining from the surrounding medium are generally low, if not negligible.

The findings from this study can be used in conjunction with analytical expressions to make preliminary predictions of the seismic response of tunnels. They can also be used as a guideline for the development of numerical models in the final design stages.

\section{Closed-form expressions for the calculation of the internal forces in a circular tunnel due to seismic wave propagation}

Of the closed-form solutions proposed for the determination of tunnel forces due to shear S-wave propagation, the most widely used are due to Wang [1]. These provide different expressions for the tunnel hoop force and the bending moment under fullslip, zero-friction conditions, as well as for the hoop force under no-slip conditions.

Under full-slip conditions at the surrounding medium-tunnel interface, the following expressions are proposed for the design hoop force $N_{\max }$ and the bending moment $M_{\max }$ of the lining:

$$
\begin{aligned}
& N_{\max }= \pm \frac{1}{6} K_{1} \frac{E_{m}}{\left(1+\nu_{m}\right)} r \gamma_{\max } \\
& M_{\max }= \pm \frac{1}{6} K_{1} \frac{E_{m}}{\left(1+\nu_{m}\right)} r^{2} \gamma_{\max }
\end{aligned}
$$


where:

$K_{1}=\frac{12\left(1-\nu_{m}\right)}{2 F+5-6 \nu_{m}}$

$F=\frac{E_{m}\left(1-\nu_{i}^{2}\right) r^{3}}{6 E_{l} l\left(1+\nu_{m}\right)} \quad=$ the flexibility ratio $[12,13]$

$\gamma_{\max }=V_{\max , S} / C_{S} \quad=$ the maximum free-field seismic shear strain due to S-wave

propagation correlated with the peak seismic velocity $\mathrm{V}_{\max , \mathrm{S}}$.

$C_{S}=\sqrt{G_{m} / \rho_{m}} \quad=$ the shear wave propagation velocity.

Under no-slip conditions at the soil-liner interface, Wang [1] proposed the following expression for the maximum hoop force:

$N_{\max }= \pm K_{2} \frac{E_{m}}{2\left(1+\nu_{m}\right)} r \gamma_{\max }$

where:

$\mathrm{K}_{2}=1+\frac{\left(1-2 v_{\mathrm{m}}\right)(1-\mathrm{C}) \mathrm{F}-0.5\left(1-2 v_{\mathrm{m}}\right) \mathrm{C}+2}{\left[\left(3-2 v_{\mathrm{m}}\right)+\left(1-2 v_{\mathrm{m}}\right) \mathrm{C}\right] \mathrm{F}+\left[0.5\left(5-6 v_{\mathrm{m}}\right)\right]\left(1-2 v_{\mathrm{m}}\right) \mathrm{C}+\left(6-8 v_{\mathrm{m}}\right)}$ and

$C=\frac{E_{m}\left(1-\nu_{1}^{2}\right) r}{E_{l} t\left(1+\nu_{m}\right)\left(1-2 \nu_{m}\right)}$ is the compressibility ratio $[12,13]$.

Using numerical analysis, the validity of this solution under specific conditions has recently been verified and discussed by Hashash et al. [10]. Note that Wang's elastic solution, as well as all other similar solutions e.g. by Penzien and Wu [2], assume infinite bond strength at the soil-structure interface. As a result, tensile stresses are allowed to develop in the liner, and the maximum tensile hoop force in the liner is algebraically equal to the maximum compressive axial force. 
Considering S-wave propagation only is a reasonable assumption in free-field conditions, i.e. for tunnels under large overburden stresses bored through homogeneous ground conditions, the shear waves will "carry" the highest portion of the seismic energy. Nevertheless, for tunnels bored through areas of irregular topographic relief or altering geotechnical conditions, the effect of secondary compressional $\mathrm{P}$-waves resulting from reflection/refraction of S-waves at the ground surface (or at the interface of soil layers with different properties) cannot be ignored [14].

In order to cover this case, Kouretzis and Andrianopoulos [14] proposed a set of closed-form expressions (Table 1), based on the work of Ranken et al. [15, 16]. These solutions follow a similar procedure to the one proposed by Wang [1] for the S-wave case.

Table 1. Internal forces of a circular liner due to P- wave propagation, under no-slip and full-slip interface conditions.

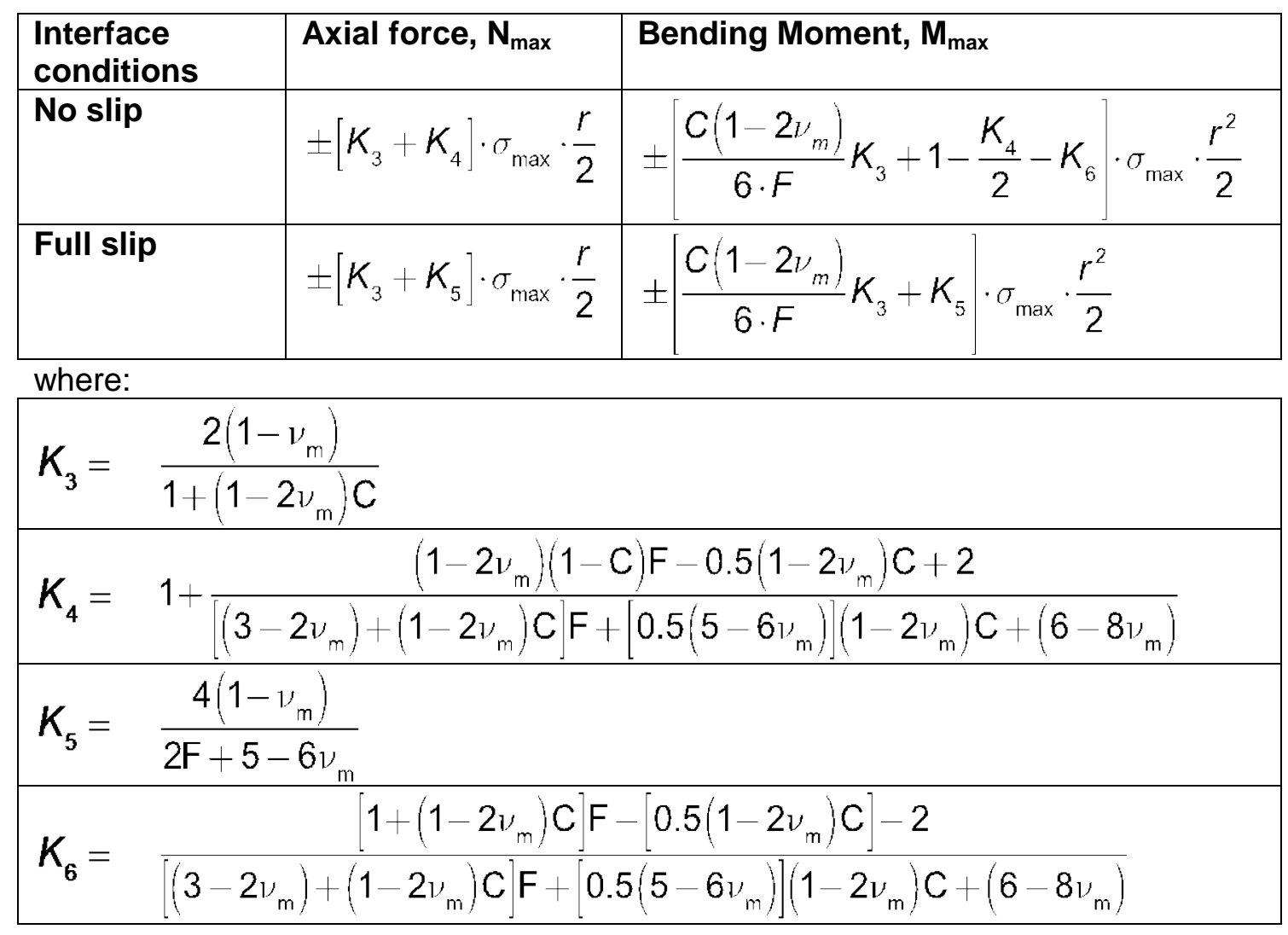


In the expressions reported in Table $1, \sigma_{\max }$ is the maximum free-field normal stress due to P-wave propagation, which is a function of the amplitude of the strong motion as well as the elastic properties of the surrounding medium:

$$
\sigma_{\max }= \pm V_{\max , P} \sqrt{\rho_{m}\left(K_{m}+\frac{4}{3} G_{m}\right)}= \pm V_{\max , P} \sqrt{\rho_{m} G_{m}} \cdot \sqrt{\frac{2\left(1-\nu_{m}\right)}{\left(1-2 \nu_{m}\right)}}
$$

In the above, the symbol ( \pm ) accounts for the cyclic nature of the loading.

Additionally, Kouretzis and Andrianopoulos [14] applied the method of Ranken et al. [15] to supplement the set of expressions proposed by Wang [1] and provide a solution for the maximum tunnel bending moment under no-slip conditions:

$$
M_{\max }= \pm\left[2-K_{4}-2 K_{6}\right] \cdot \tau_{\max } \cdot \frac{r^{2}}{2}
$$

where $T_{\max }$ is the maximum free-field seismic shear stress:

$$
\tau_{\max }= \pm V_{\max , S} \sqrt{\rho_{m} \cdot G_{m}}
$$

with the factors $\mathrm{K}_{4}$ and $\mathrm{K}_{6}$ being reported in Table 1.

As in the Wang [1] expressions, the linear elastic solution proposed by Kouretzis and Andrianopoulos [14] was derived by assuming infinite normal bond strength at the soil-tunnel contact interface. This assumption, studied numerically by Kouretzis and Andrianopoulos [14], leads to fictitious tensile hoop forces in the final lining but does not affect the compressive forces or bending moments. This effect is also reported for the S-wave case by Sedarat et al. [5], who reach similar conclusions regarding the tensile liner stresses.

\section{Numerical model and comparison of predictions with experimental results}

The numerical model to be used for the assessment of friction effects on the soilstructure interface under dynamic loads is now presented, together with a 
comparison of its predictions against the $80 \mathrm{~g}$-centrifuge observations given by Lanzano et al. [6] and Lanzano [9]. The latter describe a series of well-documented tests, simulating shallow tunnel response under seismic shear wave propagation. Bilotta et al. [8] compared the test model T1 results under earthquake excitation EQ2 $[6,9]$ against the predictions from linear dynamic numerical analyses, considering full-slip zero-friction conditions at the soil-structure interface. A sketch of the test model T1 is presented in Figure 1, while details on the centrifuge test configuration are reported in Table 2.

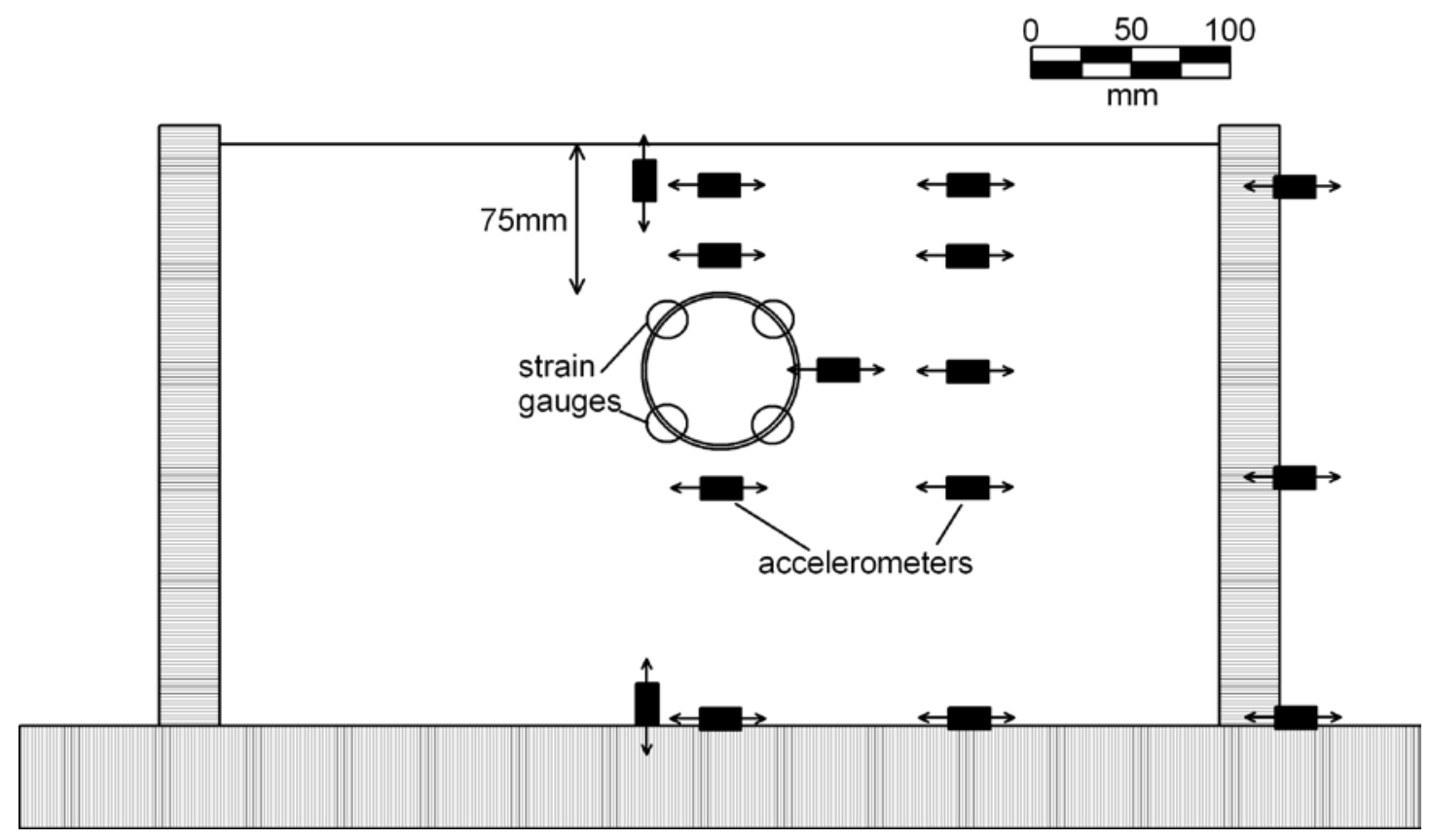

Figure 1. Simulated centrifuge test T1 configuration (after Lanzano et al. [6])

Table 2. Centrifuge test T1 - EQ2 characteristics (after Lanzano et al. [6])

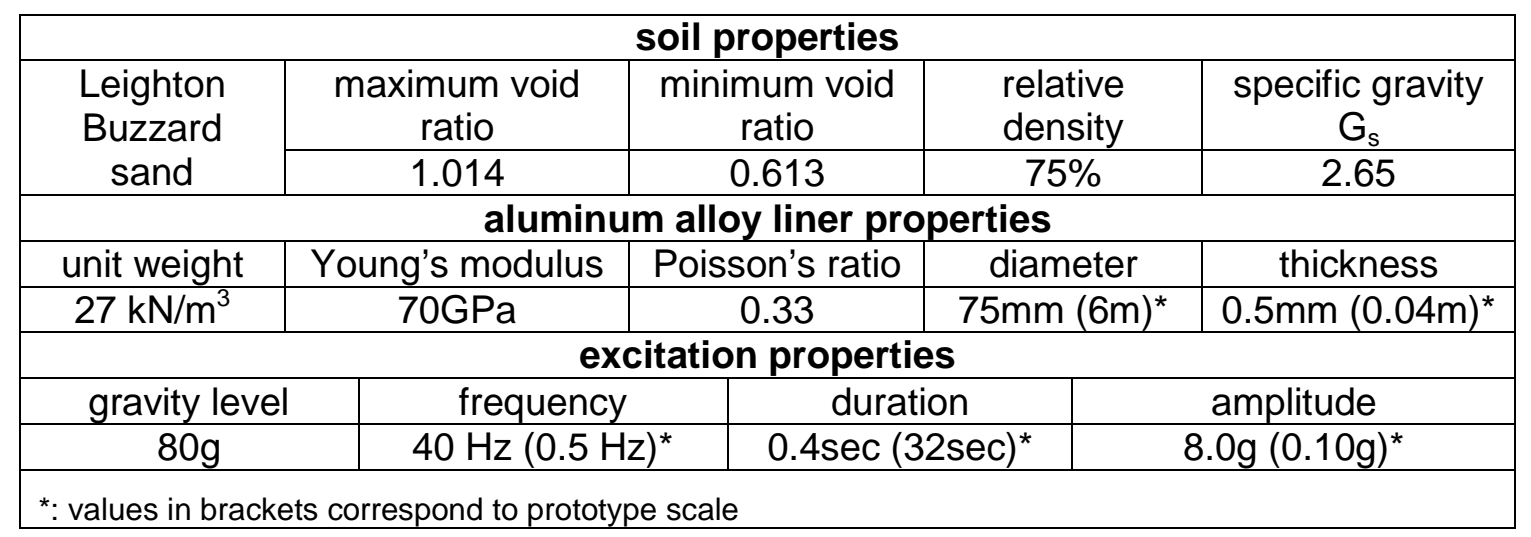




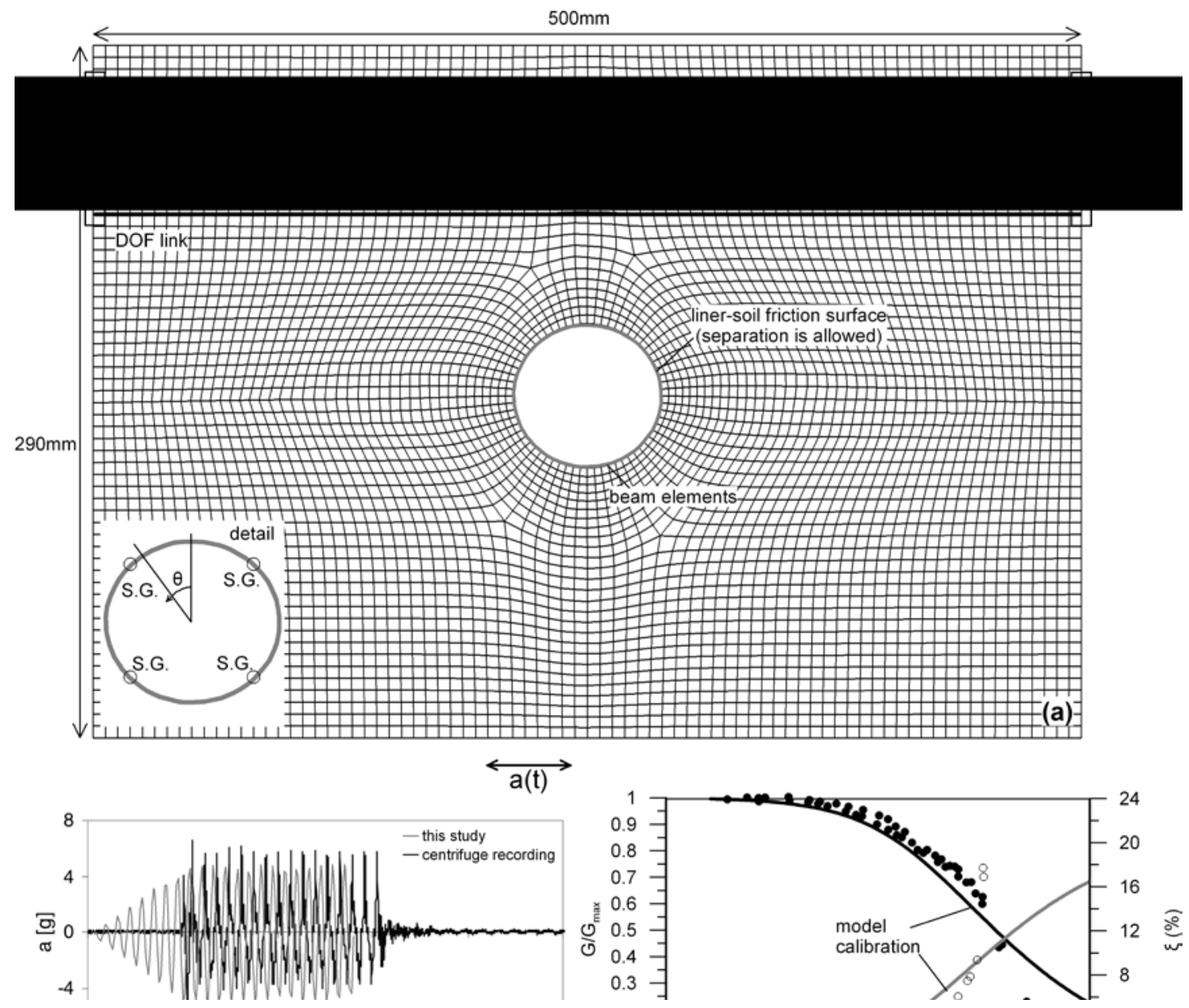

Figure 2. Numerical model for comparison with centrifuge test results (a) finite element mesh, (b) input excitation and (c) non-linear soil response together with Resonant Column data used for its calibration [24].

The numerical model presented in Figure 2 is developed in the finite element code ABAQUS/Standard to match the centrifuge test T1 by Lanzano et al. [6]. Unlike the elastic analyses presented by Bilotta et al. [8], the non-linear hysteretic response of the soil is explicitly taken into account via the implementation of an appropriate constitutive model, which is based on the Ramber-Osgood [17] model. In the adopted hypoelastic formulation, the tangential shear modulus $G_{t}$ of the soil gradually decreases from its maximum value $G_{\max }$ with increasing shear strain (black line in Figure 1c), while the opposite trend is followed by the viscous damping $\xi$ (grey line in Figure 1c). 
As described by Papadimitriou et al. [18], Papadimitriou and Bouckovalas [19], Andrianopoulos et al. [20, 21] and Karamitros [22], where the interested reader can find more details on this formulation, the tangent shear modulus $G_{t}$ is correlated to the maximum shear modulus $G_{\max }$ according to

$G_{t}=\frac{G_{\max }}{T}$

where $T \geq 1.0$ is a scalar quantifiying the reduction of the shear modulus as the current deviatoric stress ratio

$r_{i j}=\frac{\mathbf{s}_{i j}}{p}$

diverts from a reference ratio $\boldsymbol{r}_{\bar{j}}^{\text {ref }}$, where $\boldsymbol{s}_{i j}$ is the deviatoric component of stress

$\mathbf{s}_{i j}=\boldsymbol{\sigma}_{i j}-p \boldsymbol{\delta}_{i j}$

and $p$ is the isotropic stress component, i.e.

$p=\frac{\sigma_{k k}}{3}=\frac{\sigma_{11}+\sigma_{22}+\sigma_{33}}{3}$

The expression providing the scalar quantity $T$ depends on whether the soil is subjected to initial shearing ( $\notin \mathrm{LR}$ ), or subsequent load reversals ( $\in \mathrm{LR}$ ) according to $[18,19]$

$$
\mathrm{T}=\left\{\begin{array}{l}
1+2\left(\frac{1}{\alpha_{1}}-1\right)\left(\frac{\left|x_{r}\right|}{2 n_{1}}\right) \in L R \\
1+2\left(\frac{1}{\alpha_{1}}-1\right)\left(\frac{\left|x_{r}\right|}{n_{1}}\right) \notin L R
\end{array}\right.
$$

where $X_{r}$ is a scalar quantifying the variation of the current deviatoric stress ratio $\mathbf{r}_{i j}$ from the reference ratio $\boldsymbol{r}_{\bar{j}}^{\text {ref }}$ 


$$
X_{r}=\sqrt{\frac{1}{2}\left(\boldsymbol{r}_{i j}-\boldsymbol{r}_{i j}^{r e f}\right):\left(\boldsymbol{r}_{i j}-\boldsymbol{r}_{i j}^{r e f}\right)}
$$

and $\boldsymbol{r}_{i j}^{\text {ref }}$ is the deviatoric stress ratio corresponding to the point of load reversal. For the initinal shearing $\boldsymbol{r}_{\bar{j}}^{\text {ref }}$ is the initial stress ratio.

The variable $n_{1}$ in Eq. 11 is defined as

$n_{1}=\alpha_{1}\left(\frac{G_{\max }^{L R}}{p^{L R}}\right) \gamma_{1}$

where $G_{\max }^{L R}$ is the maximum shear modulus at the last load reversal, $p^{L R}$ is the isotropic stress at the last load reversal, and $\alpha_{1}, \mathrm{~V}_{1}$ are model parameters.

A shear reversal is defined when the scalar $X_{e}$ :

$$
X_{e}=\sqrt{\frac{1}{2}\left(\boldsymbol{e}_{i j}-\boldsymbol{e}_{i j}^{r e f}\right):\left(\boldsymbol{e}_{i j}-\boldsymbol{e}_{i j}^{r e f}\right)}
$$

changes sign between successive steps (i-1) and (i) i.e. when $\left[X_{e}^{(i)}-X_{e}^{(i-1)}\right]<0$. In the above Eq. (14), $\mathbf{e}_{i j}$ is the deviatoric strain tensor and $\mathbf{e}_{i j}^{\text {ref }}$ is the deviatoric strain tensor at the last shear reversal, while for the initial shearing it is the initial deviatoric strain tensor. According to Papadimitriou and Bouckovalas [19], the elastic strain increments calculated with the above equations are not fully recoverable, and the term «paraelastic» [23] would better describe such a formulation.

For the case at hand of dense Leighton Buzzard sand (Table 2), the maximum shear modulus is taken as $\mathrm{G}_{\max }=45 \mathrm{Mpa}$, according to Lanzano et al. [7], which calculated the average shear modulus mobilized during each event from top-to-base transfer functions. The model parameters $\alpha_{1}=0.80$ and $\gamma_{1}=0.0002$ are derived (Figure $1 \mathrm{c}$ ) on the basis of the experimental data from the resonant columns tests of Visone $[24,6]$. The calibration trial-and-error procedure is based on matching the analytical 
Ramberg-Osgood $G / G_{\max }-Y$ curve obtained for a certain set of $\left(\alpha_{1}, Y_{1}\right)$ parameters, to the experimental $G / G_{\max }$ data from the resonant column tests for $\gamma<\gamma_{1}$, where $\gamma_{1}$ for sands ranges between $0.0065 \%$ and $0.025 \%$ [18]. A typical stress-strain curve corresponding to the adopted parameters is presented in Figure 3.

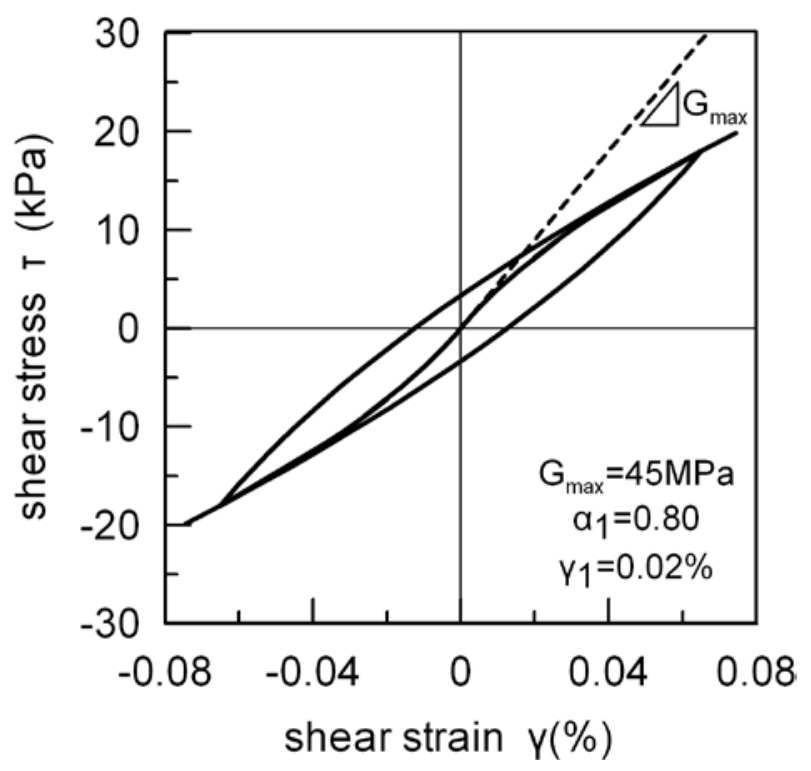

Figure 3. Typical Ramberg-Osgood stress-strain curve, for the model parameters used in the numerical analyses.

The Poisson's ratio of the soil is taken as $v_{m}=0.333$, while its density is equal to $\rho_{\mathrm{m}}=1.55 \mathrm{Mg} / \mathrm{m}^{3}$. No Rayleigh damping is introduced in the model, with the only source of material damping being energy dissipation from the hysteretic soil response.

The analyses are executed at model scale, with the aluminum alloy tube modeling the tunnel liner properties presented in Table 2. Beam elements are used for its simulation, while in order to account for plane strain conditions the Young's modulus of the alloy is divided by $\left(1-\nu_{i}^{2}\right)$. The tunnel embedment depth in the model scale is $75 \mathrm{~mm}$ (Figure 1).

The numerical analyses consist of two steps: The first, a static (swing-up) step, includes the application of a gravitational force equal to $80 \mathrm{~g}$, which is held constant 
during the subsequent dynamic step, where the seismic excitation is applied. During the first analysis step, a linear elastic model with shear modulus $G=G_{\max }$ is employed for the calculation of the gravity stress distribution, while the bottom boundary of the model is fixed in the vertical direction, and the lateral boundaries of the model are fixed in the horizontal direction.

In the subsequent dynamic analysis step, the input sinusoidal excitation (shown in Figure $1 \mathrm{~b}$ ) is applied at the fixed base of the model, and features a frequency $f=40 \mathrm{~Hz}$ and a maximum amplitude $a=5 \mathrm{~g}$, as well as an initial ramp section of 8 periods to eliminate the need for baseline correction. The excitation parameters are selected to match as accurately as possible the input excitation presented by Bilotta et al. [8] since the latter was not available in digital format. The centrifuge input excitation is compared with the applied excitation in Figure $1 b$.

To account for deformation of the laminar box, the vertical boundaries of the mesh are connected via rigid links, while the bottom boundary is fixed. The contact between the sand and the aluminium alloy tube allows for separation, while different assumptions are adopted for the penalty friction formulation used to model the tangential behavior of the interface: In the first analysis, zero friction is considered following Bilotta et al. [8]. In the second and third analyses, coefficients of friction $\mathrm{n}=0.1$ and $\mathrm{n}=0.5$ are considered, which correspond to friction angles at the soil-tube interface equal to $\delta=5.7^{\circ}$ and $\delta=26.5^{\circ}$, respectively. The linear penalty method is used to simulate the normal surface behavior, together with an efficient iterative procedure that minimizes penetration of surfaces (Augmented Lagrange method [11]).

Figure 5 compares the peak ground accelerations from the numerical analyses with those from the centrifuge test measurements, as well as the numerical results presented by Bilotta et al. [8]. The difference in the peak value of the input 
acceleration at the bottom is due to the exclusion of the high-frequency component at the beginning of the experimental measurement of the acceleration. This feature does not affect the results higher in the sample since the high-frequency component is filtered by the soil.

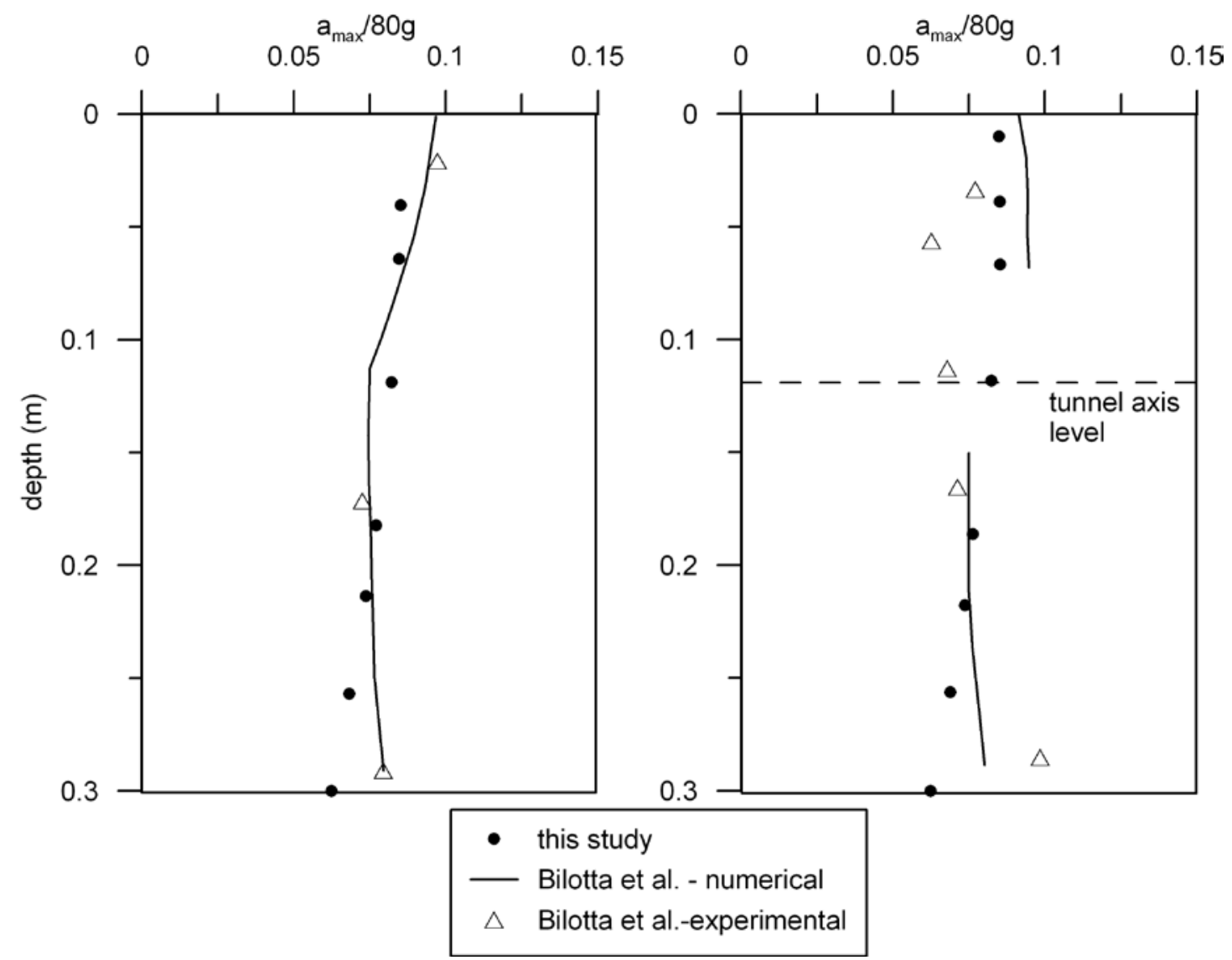

Figure 4. Comparison of peak ground accelerations (a) at the free-field, and (b) along the tunnel vertical axis.

Tables $3 a$ and $3 b$ compare the peak axial (hoop) forces and bending moments predicted from the numerical analyses against the centrifuge test. These results are at the polar angles $\theta$ along the tube periphery where strain gages (SG) were installed (see Figures 1 and 2a). As in Bilotta et al. [8], the average values of the peak-to-peak amplitude for axial forces and bending moments are used to compute the seismic increments $\Delta N$ and $\Delta M$, while the steady-state part of the seismic motion is considered when the acceleration has reached its peak value (Figure 5). 
Table 3a. Comparison of numerical against experimental results, in terms of dynamic axial hoop forces.

\begin{tabular}{|l|l|l|l|}
\hline maximum axial hoop force & \multicolumn{3}{|c|}{ location along the cross-section } \\
\cline { 2 - 4 } dynamic increment, $\mathbf{N}_{\max }(\mathbf{N} / \mathbf{m m})^{*}$ & $\boldsymbol{\theta = 1 3 5 ^ { \circ }}$ & $\boldsymbol{\theta = 2 2 5 ^ { \circ }}$ & $\boldsymbol{\theta = 3 1 5 ^ { \circ }}$ \\
\hline centrifuge & 0.0025 & 0.0050 & 0.0047 \\
\hline numerical, $\mathbf{n = 0}$ & 0.0065 & 0.0067 & 0.0075 \\
\hline numerical, $\mathbf{n = 0 . 1}$ & 0.0686 & 0.0683 & 0.0442 \\
\hline numerical, $\mathbf{n = 0 . 5}$ & 0.2206 & 0.2364 & 0.1520 \\
\hline \multirow{2}{*}{$*$ values are presented as N per running mm of liner } \\
\hline
\end{tabular}

Table 3b. Comparison of numerical against experimental results, in terms of dynamic bending moments.

\begin{tabular}{|l|l|l|l|l|}
\hline \multirow{2}{*}{$\begin{array}{l}\text { maximum bending moment dynamic } \\
\left.\text { increment, } \mathbf{M}_{\max } \mathbf{( N m m} / \mathbf{m m}\right)\end{array}$} & \multicolumn{4}{|c|}{ location along the cross-section } \\
\cline { 2 - 5 } & $\boldsymbol{\theta = \mathbf { 4 5 } ^ { \circ }}$ & $\boldsymbol{\theta = 1 3 5 ^ { \circ }}$ & $\boldsymbol{\theta = 2 2 5 ^ { \circ }}$ & $\boldsymbol{\theta = 3 1 5 ^ { \circ }}$ \\
\hline centrifuge & 0.030 & 0.045 & 0.075 & 0.085 \\
\hline numerical, $\mathbf{n = 0}$ & 0.030 & 0.046 & 0.034 & 0.033 \\
\hline numerical, $\mathbf{n = 0 . 1}$ & 0.029 & 0.044 & 0.033 & 0.029 \\
\hline numerical, $\mathbf{n = 0 . 5}$ & 0.026 & 0.040 & 0.032 & 0.027 \\
\hline$*:$ values are presented as Nmm per running mm of liner \\
\hline
\end{tabular}



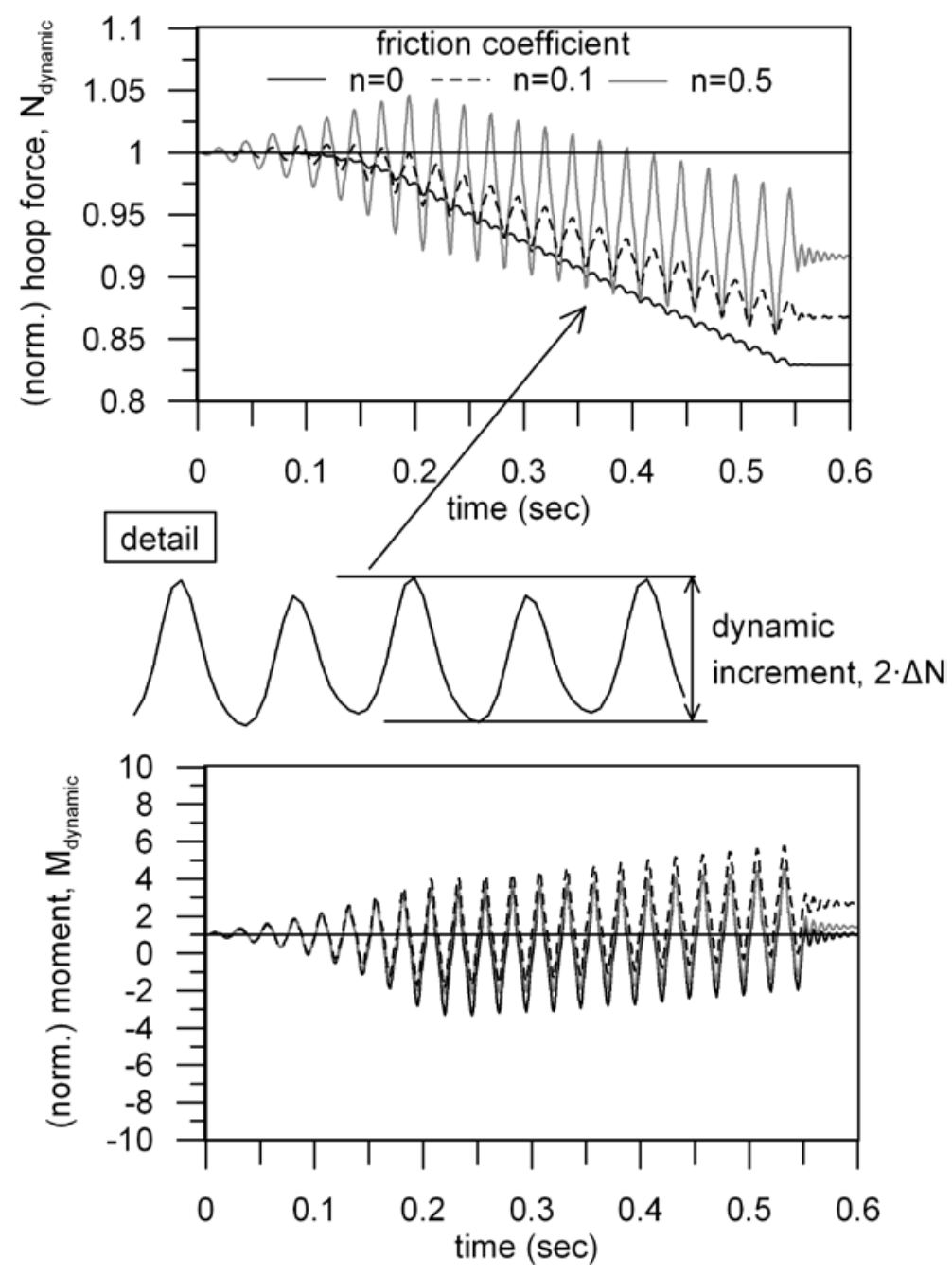

Figure 5. Liner hoop forces and bending moment time histories at $\theta=315^{\circ}$, normalized against their value at the end of the static step. Results for three different friction coefficient assumptions at the liner-sand interface are presented.

The complete time-histories of the hoop force and bending moment at the polar angle $\theta=315^{\circ}$ are presented in Figure 5 for the three different interface friction coefficients. Forces and moments are normalized against their value at the end of the static analysis step, to obtain their dynamic component $N_{d y n a m i c}$ and $M_{d y n a m i c}$, respectively, as the dynamic liner response is of interest here.

Notice that there is a clear shift in the internal liner forces developing throughout the duration of excitation, associated with non-recoverable deformation of the liner. This type of behaviour is attributed to the fact that as the shear modulus of the soil 
reduces with increasing shear strain, a re-distribution of stresses takes place causing deformation of the tube, measured as heave of the liner crown (Figure 5). This is due to the extremely flexible liner used in centrifuge tests to obtain accurate dynamic strain measurements [6], which features a diameter-to-thickness ratio $D / t=150$. Such a flexible liner cannot contribute singificant support to the surrounding sand, and the system essentially behaves like a medium with a loosely supported void.

To further substantiate that, an additional analysis is performed, considering a liner with a diameter-to-thickness ratio $\mathrm{D} / \mathrm{t}=15-\mathrm{a}$ more realistic scenario, which corresponds to a tunnel with diameter $D=6 \mathrm{~m}$ and liner thickness $t=0.4 \mathrm{~m}$ in prototype scale. As it is depicted from Figure 6, in that case this effect is eliminated. A shift in axial force and bending moment was also measured experimentaly, and it was attributed by Lanzano et al. [6] to soil densification, as the void ratio of the sand is reduced from $\mathrm{e}=0.71$ to $\mathrm{e}=0.68$ after the completion of the test $\mathrm{T} 1$; and could be considered as an indication of the insufficinent support provided by the liner. The hypoelastic model used in this study cannot capture soil densification effects, and the shift measured during centrifuge tests cannot be addressed. However, such an effect not important for the problem examined herein, where we focus on the transient component of internal force increments. 

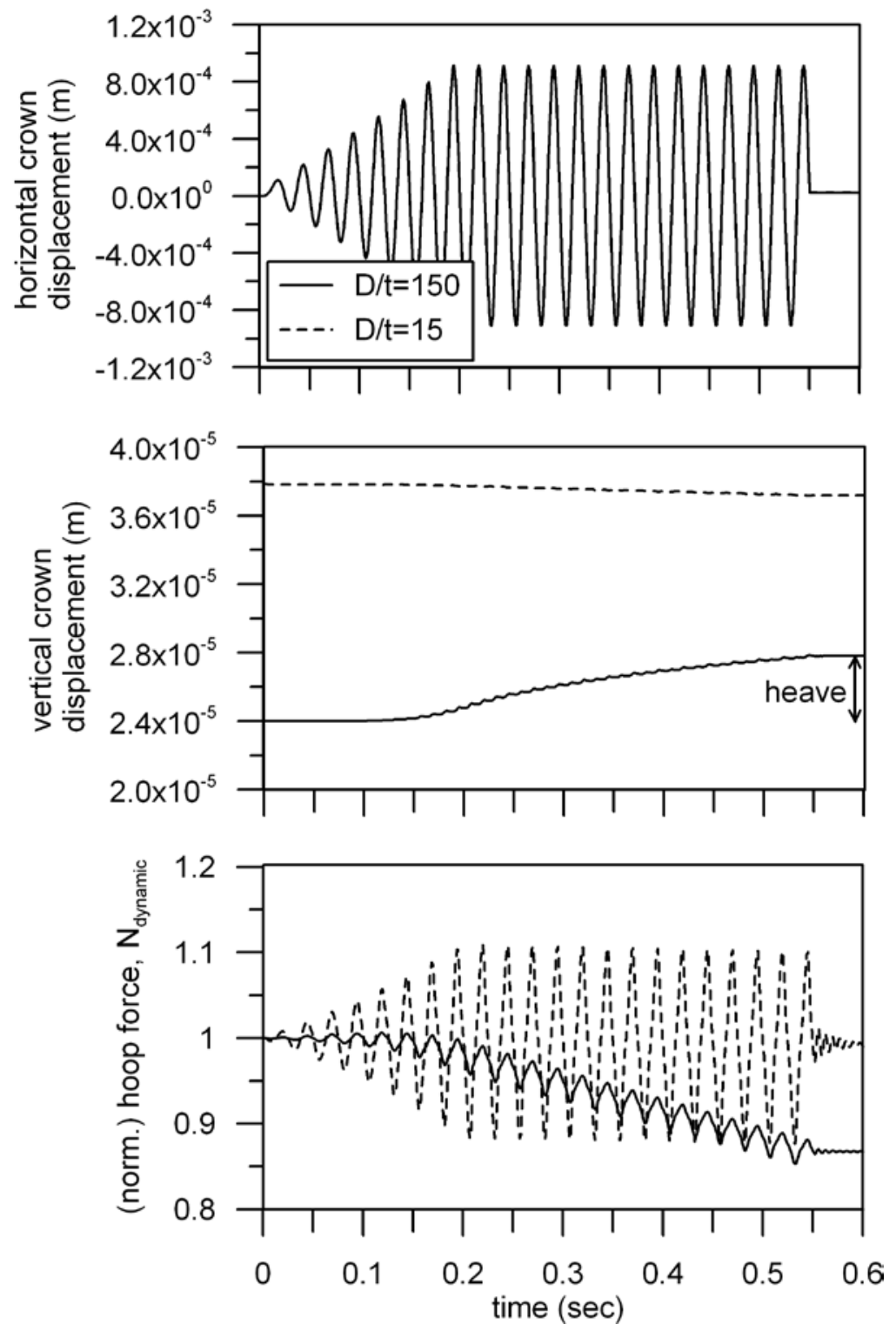

Figure 6. Axial hoop force time histories at $\theta=315^{\circ}$, and liner crown displacements, for a very flexible $(D / t=150)$ and a stiffer $(D / t=15)$ liner. Analyses for friction value $\mathrm{n}=0.1$; displacement results are presented at model scale.

The best match to the centrifuge test results is achieved when a zero friction condition at the sand-tube interface is assumed, as in the numerical analyses presented by Bilotta et al. [8]. These authors considered an elastic soil model, and incorporated Rayleigh damping to simulate soil hysteretic behavior, yet their results are compatible with the results of the non-linear analyses presented here and the centrifuge test results. As the friction coefficient increases, a rapid increase in the dynamic portion of the axial forces is observed (Figure 5, Table 3), while the bending moments are much less affected. This conclusion is consistent with the findings of 
Sedarat et al. [5] for tunnels with similar flexibility factors, F. It is interesting to mention that even when a very low friction value is introduced at the interface, the axial hoop force values diverge grossly from the centrifuge test results (Figure 5, Table 3). This divergence occurs for friction angles which are much lower than $\tan \delta=\tan (0.7 \varphi)-$ the recommended friction angle for a cohesionless soil-smooth steel interface according to ASCE-ALA [25] guidelines for axial static pipeline movement relative to surrounding soil.

\section{Parametrical investigation of friction effects on internal forces}

In order to parametrically assess the effect of friction on the tunnel internal forces, a simple numerical model is developed. This model, presented in Figure 7, simulates shear and compressional wave propagation in an elastic space, i.e. it is compatible with the assumptions of the analytical solutions by Wang [1] and Kouretzis and Andrianopoulos [14]. Infinite elements, functioning as absorbing boundaries, are incorporated at the top and bottom boundaries of the model to avoid wave reflection phenomena, while lateral boundaries are not restrained. The input sinusoidal motion, with the characteristics presented in Figure 6, is applied at the bottom of the model as a stress time history. The direction of the input stress varies, depending on the wave type modelled (shear or compressional).

Firstly, a set of four basic analyses is performed in order to compare the results of the numerical model with the analytical closed-form expressions presented in paragraph 2. The properties of the model are summarized in Table 4. The four analyses simulate the tunnel response under S- and P-wave propagation: first under full-slip conditions at the soil-structure interface, assuming zero-friction and allowing for separation under tensile contact stresses, and second under no-slip conditions, where the beam elements of the liner are tied to the nodes of the surrounding soil. These two extreme cases correspond to the assumptions under which the analytical 
expressions were derived. For comparison purposes, the hoop forces and bending moments are normalized as $\mathrm{N} /(\sigma \cdot r)$ and $\mathrm{M} /\left(\sigma \cdot r^{2}\right)$ respectively [1], with $\sigma$ being the maximum seismic normal stress for P-waves. This latter value is replaced by the maximum seismic shear stress, $\mathrm{T}$, for the case of shear S-waves.

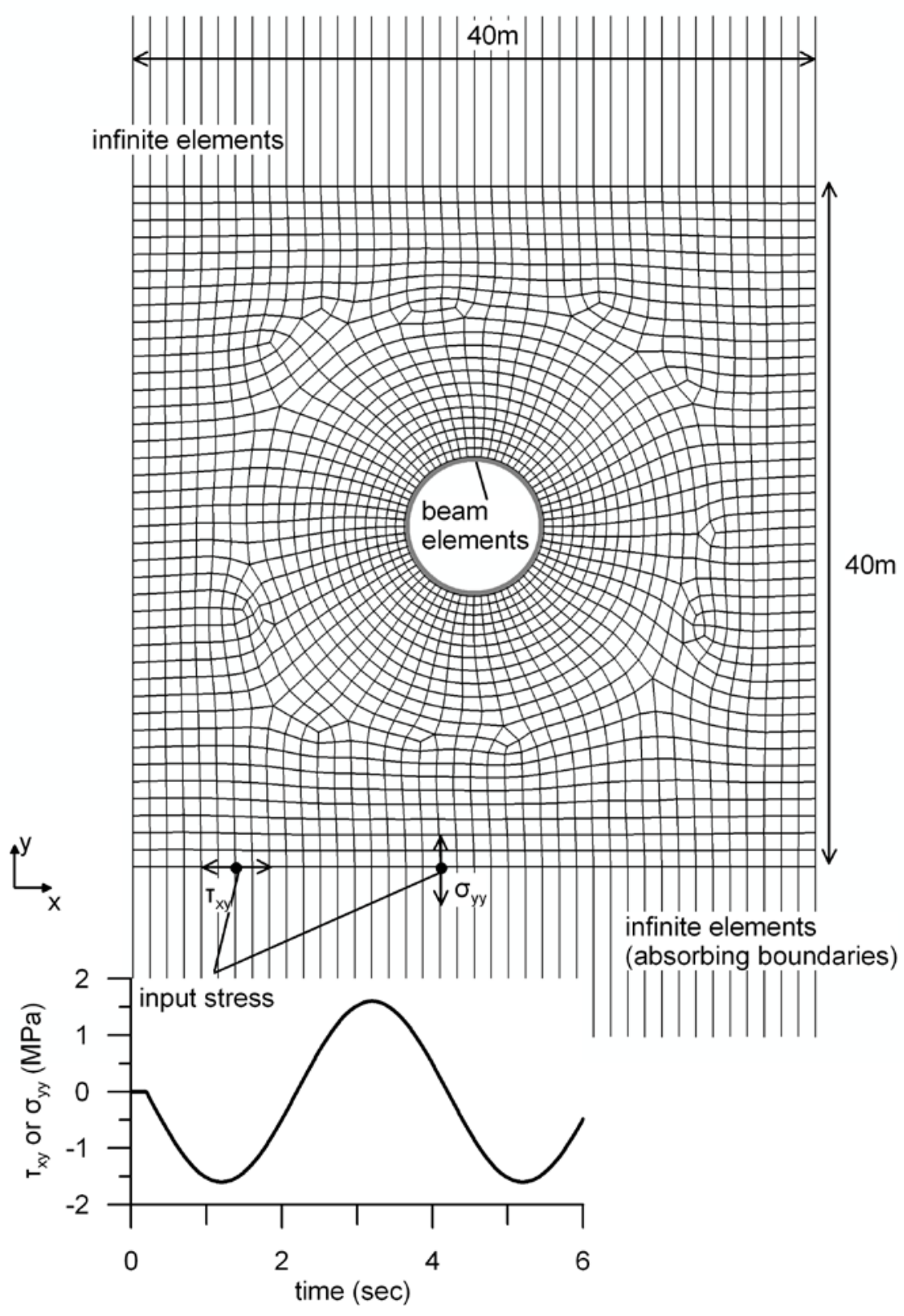

Figure 7. Finite element mesh to model seismic wave propagation in an infinite elastic medium. 
Table 4. Input data of the basic set of analyses (Case 1).

\begin{tabular}{|l|l|}
\hline \multicolumn{2}{|l|}{ medium properties } \\
\hline Modulus of elasticity, $\mathrm{E}_{\mathrm{m}}$ & $2 \mathrm{GPa}$ \\
\hline Poisson ratio, $\mathrm{v}_{\mathrm{m}}$ & 0.2 \\
\hline liner properties & \\
\hline Modulus of elasticity, $\mathrm{E}_{\mathrm{I}}$ & $19 \mathrm{GPa}$ \\
\hline radius, $\mathrm{r}$ & $4.0 \mathrm{~m}$ \\
\hline Poisson ratio, $\mathrm{v}_{\mathrm{I}}$ & 0.2 \\
\hline thickness, $\mathrm{t}$ & $0.15 \mathrm{~m}$ \\
\hline Flexibility ratio & 3193 \\
\hline Compressibility ratio & 3.74 \\
\hline
\end{tabular}

The results presented in Figure 8 show a fair agreement between the analytical and numerical maximum values. Some numerical noise is introduced in the P-wave bending moment time history during the separation phase, without significantly affecting the solution (Figure 8). Observe that the axial forces due to S-wave propagation under full-slip conditions are practically zero for this 'flexible' tunnel (with a flexibility factor of $F=3193$ ) while, when separation is possible at the soil-structure interface, no tensile forces develop in the liner. The latter finding is in line with the results presented by Sedarat et al. [5] and Kouretzis and Andrianopoulos [14]. It is also compatible with the centrifuge observations of Lanzano [9], who commented that '...the hoop forces are generally always positive, except some case(s) of null or slightly negative value(s).' 

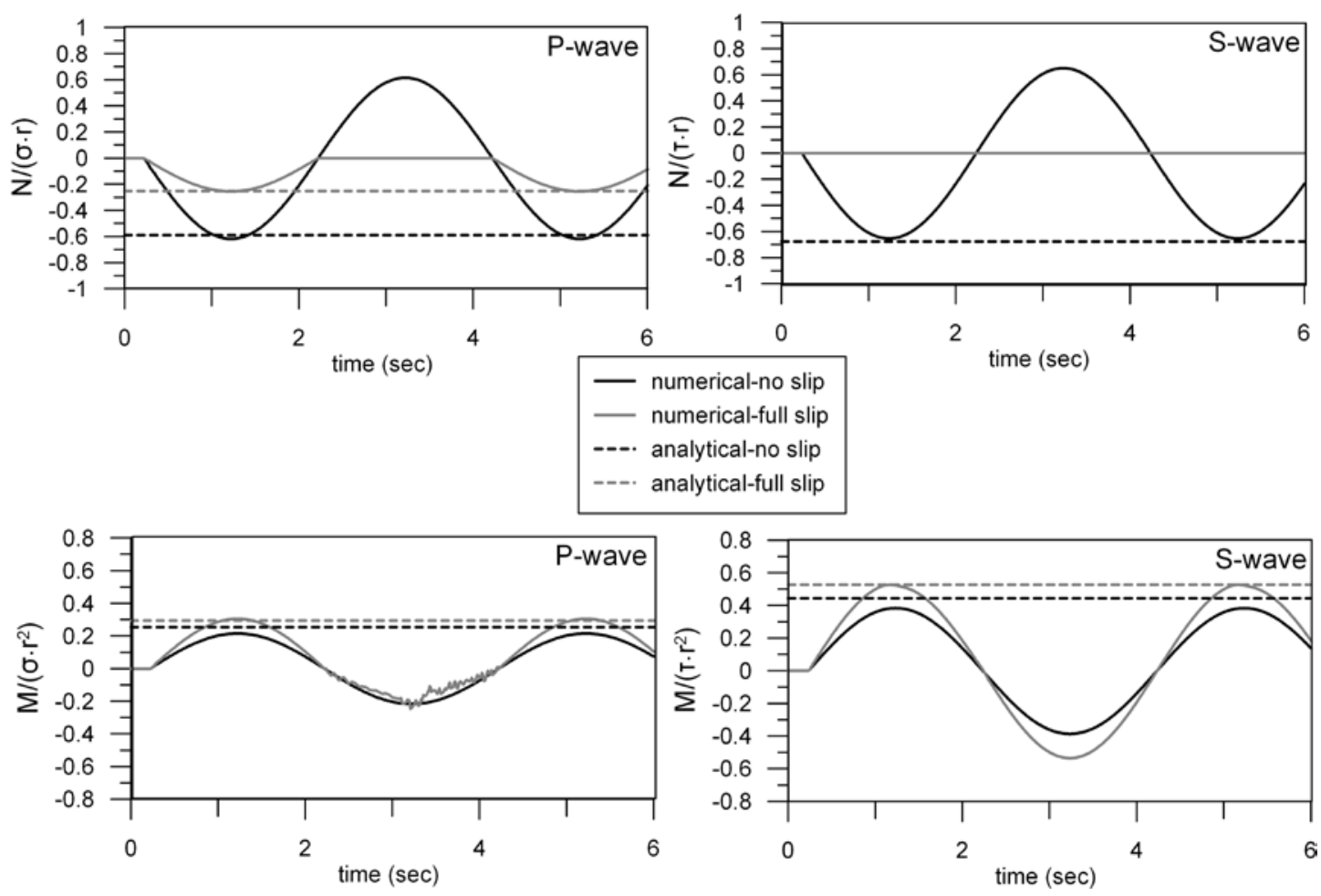

Figure 8. Comparison of analytical with numerical results from the basic analyses set (Case 1). Negative hoop force values correspond to compressional liner normal stresses.

To investigate the effect of friction at the soil-tunnel interface, a series of parametric analyses are performed for both the S- and the P-wave scenarios, considering three cases. Case 1 corresponds to a 'flexible' tunnel (flexibility factor $F=3193$, compressibility factor $C=3.74$ ) with the properties presented in Table 4 . Cases 2 and 3 are for stiffer tunnels, with $F=319 / C=0.37$ and $F=31.9 / C=0.037$, respectively. These effectively cover the whole range of flexibility and compressibility factors for typical tunnels. This variation is achieved by reducing the surrounding soil Young's modulus in each analysis to attain the required factors. No gravitational forces are acting on the model, thus the forces on the liner from the soil loads were equal to zero. 

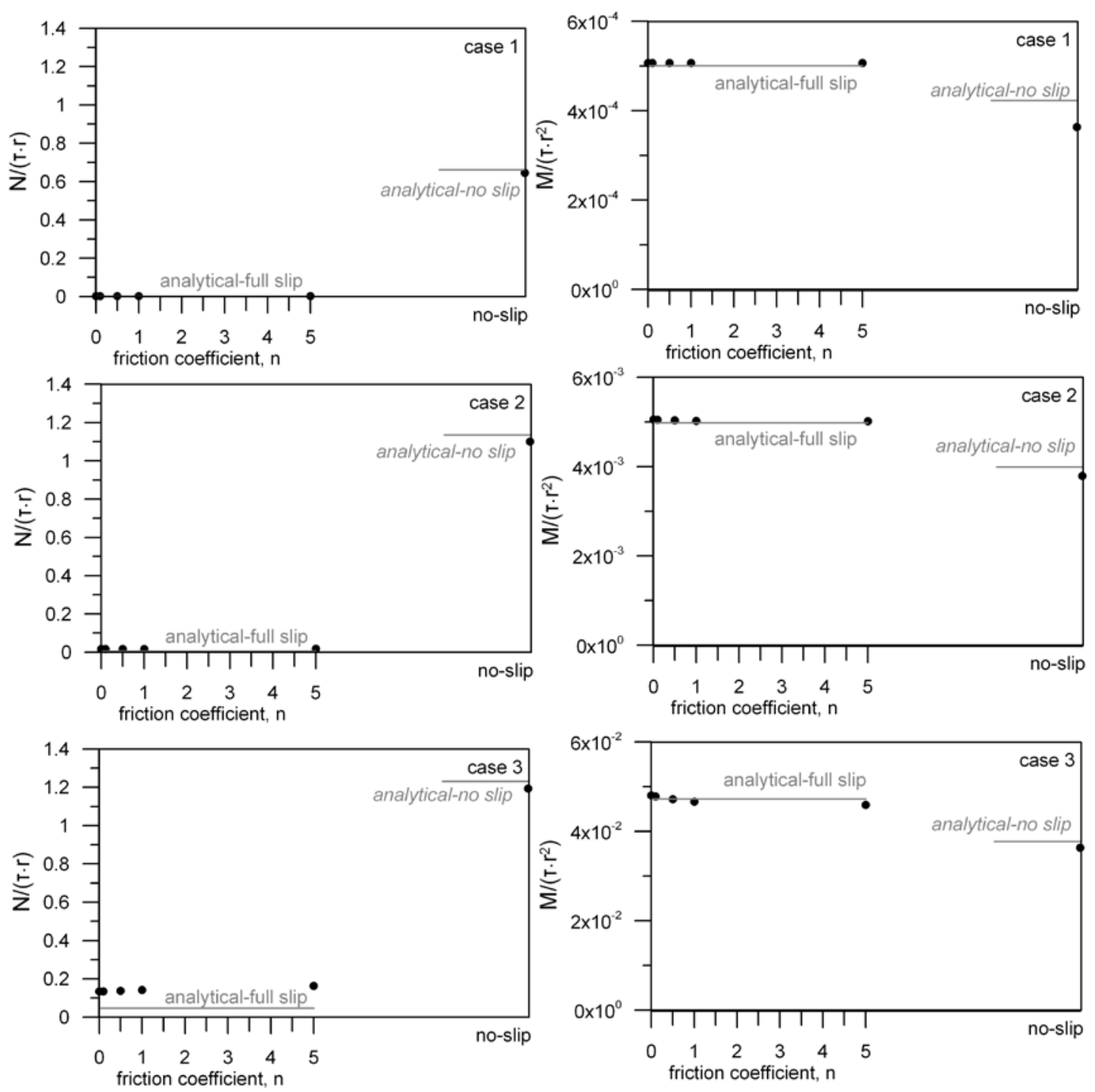

Figure 9. Results of the parametric analyses for the S-wave case. Absolute maximum values of normalized internal forces are presented.

For each case five (5) different values of the friction coefficient are considered, ranging from zero up to a very high value of $n=5$, while free separation at the soiltunnel interface under tensile normal stresses is allowed by default. An additional analysis is performed for each case, considering no-slip conditions and perfect bond at the interface. The results from the numerical analyses are compared with the corresponding analytical solutions in Figures 9 and 10. 

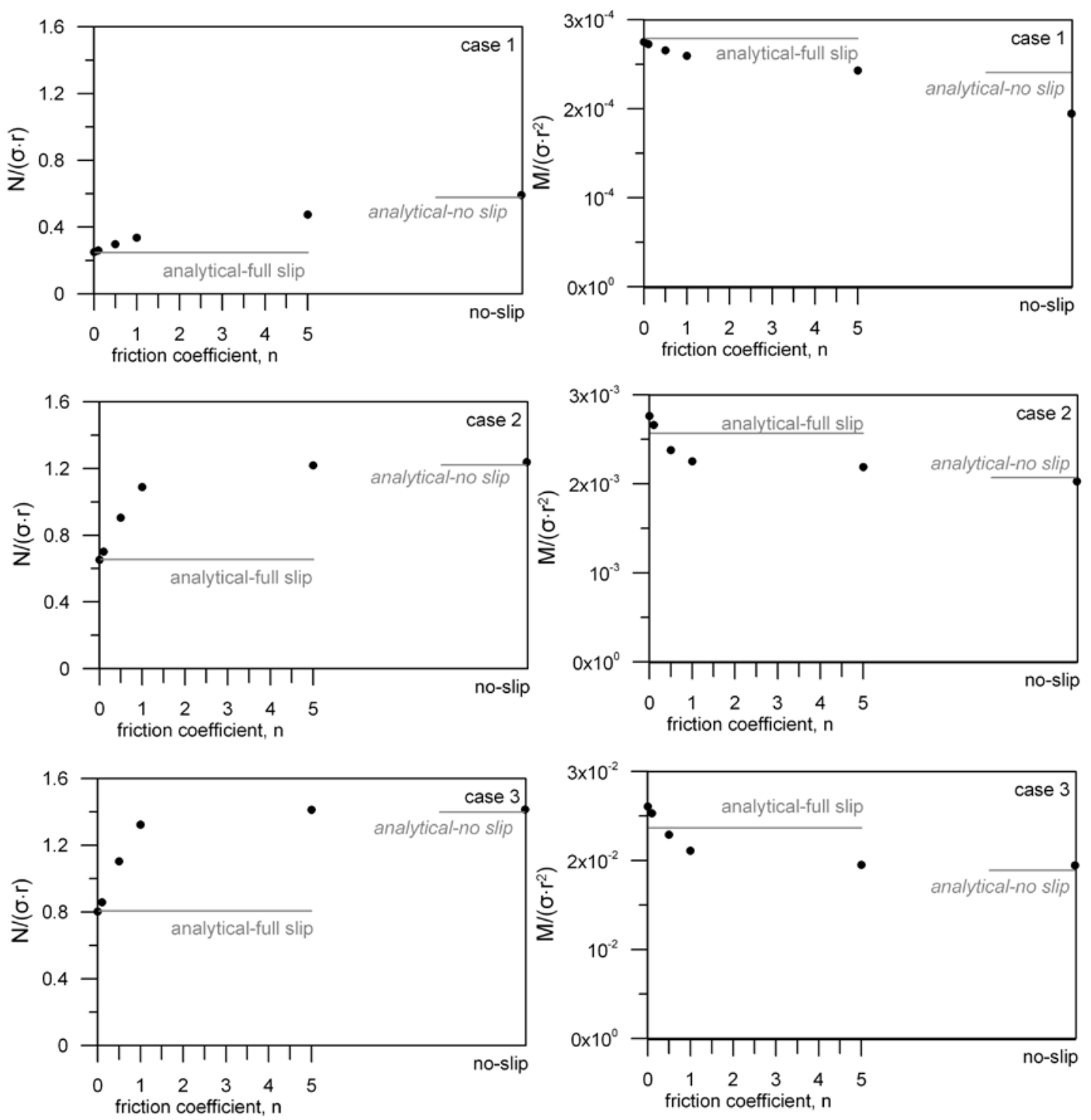

Figure 10. Results of the parametric analyses for the P-wave case. Absolute maximum values of normalized internal forces are presented.

As shown in Figure 9, the effect of friction on the axial forces and bending moments for the S-wave case is trivial when the load of the soil mass is negligible. This is due to the fact that compressive normal stresses at the contact interface are zero. This finding is significant for the axial liner force, since the difference between the cases where full-bond is assumed is dramatic. Notice that there is a slight divergence between analytical and numerical axial liner forces from the S-wave Case 3 analyses (Figure 9). This is due to the fact that full-slip axial forces due to S-wave propagation are very low, practically zero, and a similar relative divergence for cases 1 and 2 is not depicted in Figure 9, due to the scale of the vertical axis. 
On the other hand, due to the different mode of deformation imposed by P-wave loading, where normal stresses are applied at the soil-lining interface as a result of propagation of the seismic wave train, the transition of the internal forces from the full-slip case to the no-slip case is rather smooth. Indeed, as the friction coefficient increases, the numerical results tend to match the no-slip case results closely. However, the increase in the axial liner forces for realistic values of $n(n<0.5)$ is gradual, at least for 'flexible' tunnels. The opposite effect occurs for the bending moments, where the maximum values tend to decrease with increasing friction at the interface.

\section{Concluding remarks and practical implications}

The effect of the soil-lining interface properties on the internal forces developed in the lining of a circular tunnel due to seismic loading was investigated numerically, for both (shear) S- and (compressional) P-wave propagation. The finite element results were validated against centrifuge test measurements and existing closed-from elastic solutions. The focus of the parametric part of this study was on circular linings not carrying any loads from the soil mass, an assumption which is valid for unreinforced tunnel final linings where the relaxation load due to tunnel excavation is assumed to be transferred only to the temporary support of the tunnel. Practical findings of this study can be summarized as follows:

Comparison of non-linear dynamic numerical analyses with the centrifuge test results presented by Lanzano et al. [6] suggest that test friction conditions at the dense sand-liner interface are close to the zero-friction assumption. If loads are transferred directly to the tunnel final lining from the surrounding soil, introducing even a very low friction value in the numerical interface simulation results in grossly higher axial forces. 
- When the loads of the surrounding soil mass are assumed to be borne by the temporary support shell, the effect of friction angle on the structural response of the lining is negligible for the S-wave case, since the normal stresses at the interface are zero. This is not the case when compressional P-waves are considered in the analysis, e.g. for tunnels bored through irregular topography on through non-uniform geological formations, although for realistic friction values this effect is not significant.

- $\quad$ For unreinforced tunnel final linings, when no soil mass loads are generally expected to be transferred to the final lining and the main failure mode in the final lining is the development of intolerable cracks due to high eccentricity of the hoop force, the closed-form analytical solutions provide reasonable estimates of the liner forces under the full-slip condition. However, for the noslip, full-bond interface condition, the results give unrealistically high axial hoop forces. These solutions would also not yield realistic results when the final lining is designed to bear the surrounding soil loads (e.g. TBM tunnels, or even buried pipelines). The selection of the proper interface friction angle is an issue that deserves further experimental investigation.

\section{References}

[1] Wang J.N., 1993. Seismic design of tunnels-A simple state-of-the-art design approach. Parsons Brinckerhoff, New York, Monograph 7.

[2] Penzien J. and Wu C.L., 1998. Stresses in linings of bored tunnels. Earthquake Engineering and Structural Dynamics. 27, 283-300.

[3] Park K.-H., Tantayopin K., Tontavanich B. and Owatsiriwong A., 2009. Analytical solution for seismic-induced ovaling of circular tunnel lining under no-slip interface conditions: A revisit. Tunneling and Underground Space Technology. 24, 231-235.

[4] Hashash Y.M.A., Hook J.J., Schmidt B., Yao J.I-C., 2001. Seismic design and analysis of underground structures. Tunneling and Underground Space Technology. 16, 247-293. 
[5] Sedarat H., Kozak A., Hashash Y.M.A., Shamsabadi A. and Krimotat A., 2009. Contact interface in seismic analysis of circular tunnels. Tunneling and Underground Space Technology. 24, 482-490.

[6] Lanzano G., Bilotta E., Russo G., Silvestri F. and Madabhushi S.P.G., 2012. Centrifuge Modeling of Seismic Loading on Tunnels in Sand. Geotechnical Testing Journal 35(6).

[7] Lanzano G., Bilotta E., Russo G., Silvestri F. and Madabhushi S.P.G., 2010. Dynamic centrifuge tests on shallow tunnel models in dry sand. 7th International Conference on Physical Modelling in Geotechnics, ICPMG, pp. 561-567.

[8] Bilotta E., Lanzano G., Russo G., Silvestri F. and Madabushi G., 2009. Seismic analyses of shallow tunnels by dynamic centrifuge tests and finite elements. Proceedings of the $17^{\text {th }}$ International Conference on Soil Mechanics and Geotechnical Enigneering. M. Hamza et al. (Eds.) IOS Press.

[9] Lanzano G., 2009. Physical and analytical modeling of tunnels under dynamic loadings. PhD thesis. University of Naples "Federico II".

[10] Hashash Y.M.A., Park D., Yao J.I-C., 2006. Ovaling deformations of circular tunnels under seismic loading, an update on seismic design and analysis of underground structures. Tunneling and Underground Space Technology. 20, 435441.

[11] Abaqus/Standard - User's Manual - version 6.11. Dassault Systemes Simulia Corp.

[12] Hoeg, K. 1968. Stresses against underground structural cylinders. Journal of Soil Mechanics and Foundation Division ASCE, 94:SM4.

[13] Peck, R.B., Hendron, A.J., Mohraz, B., 1972. State of the art in soft ground tunneling. In: The Proceedings of the Rapid Excavation and tunneling Conference. American Institute of Mining, Metallurgical and Petroleum Engineers, New York, NY, pp. 259-286.

[14] Kouretzis G.P. and Andrianopoulos K.A., 2012. On the aseismic analysis of circular tunnels, with emphasis on unreinforced concrete liners. Tunneling and Underground Space Technology (submitted for publication).

[15] Ranken, R.E., Ghaboussi, J., Hendron A.J., 1978. Analysis of ground-liner interaction for tunnels. Report No. UMTA-IL-06-0043-78-3, U.S. Department of Transportation, Washington D.C.

[16] F.H.W.A., 2009. Technical manual for design and construction of road tunnels-Civil elements. U.S. Department of Transportation, Federal Highway Administration. Publication No. FHWA-NHI-10-034, December.

[17] Ramberg W. and Osgood W.R., 1943. Description of stress-strain curves by three parameters. Technical Note 902, National Advisory Committee for Aeronautics, Washington D.C.

[18] Papadimitriou A.G., Bouckovalas G.D. and Dafalias Y.F., 2001. Plasticity model for sand under small and large cyclic strains. Journal of Geotechnical and Geoenvironmental Engineering. 127 (11), 973-983. 
[19] Papadimitriou A.G. and Bouckovalas G.D., 2002. Plasticity model for sand under small and large cyclic strains: a multiaxial formulation. Soil Dynamics and Earthquake Engineering. 22 (3), 191-204.

[20] Andrianopoulos K. I., Papadimitriou A. and Bouckovalas G., 2010. Explicit integration of bounding surface model for the analysis of earthquake soil liquefaction. International Journal for Numerical and Analytical Methods in Geomechanics. 34 (15), 1586-1614.

[21] Andrianopoulos K. I., Papadimitriou A. G. and Bouckovalas G. D., 2010. Bounding surface plasticity model for the seismic liquefaction analysis of geostructures. Soil Dynamics and Earthquake Engineering. 30 (10), 895-911.

[22] Karamitros D., 2010. Development of a numerical algorithm for the dynamic elastoplastic analysis of geotechnical structures in two (2) and three (3) dimensions. PhD thesis. National Technical University of Athens, Greece.

[23] Hueckel T. and Nova R., 1979. Some hysteresis effects of the behavior of geological media. Int J Solids Struct. 15: 625-642.

[24] Visone C., 2008. Performance based design of embedded retaining walls. PhD Thesis. University of Naples "Federico II".

[25] American Lifelines Alliance ALA, 2005. Guidelines for the design of buried steel pipes. New York: ASCE.

\section{Nomenclature}

$\mathrm{M} \quad$ lining bending moment

$\mathrm{N} \quad$ lining axial hoop force

$E_{m} \quad$ elasticity modulus of the surrounding medium

$E_{1} \quad$ elasticity modulus of the tunnel liner

$v_{m} \quad$ Poisson ratio of the surrounding medium

$v_{l} \quad$ Poisson ratio of the tunnel liner

$r$ tunnel radius

D tunnel diameter

$\mathrm{t} \quad$ liner thickness

I moment of inertia of the tunnel liner

$Y_{\max } \quad$ maximum free-field shear strain

$\mathrm{G}_{\mathrm{m}} \quad$ shear modulus of the surrounding medium

$\mathrm{K}_{\mathrm{m}} \quad$ bulk modulus of the surrounding medium

$\mathrm{G}_{\max }$ maximum soil shear modulus

$\mathrm{G}_{\mathrm{t}} \quad$ tangent soil shear modulus

$\rho_{m} \quad$ density of the surrounding medium

$\mathrm{V}_{\text {max,s }}$ peak seismic velocity due to shear wave propagation 
$\mathrm{V}_{\text {max,P }}$ peak seismic velocity due to compressional wave propagation

$F \quad$ flexibility ratio

C compressibility ratio

$\mathrm{Vm}_{\mathrm{m}} \quad$ unit weight of the surrounding medium

h tunnel overburden.

$\mathrm{K}_{\mathrm{o}} \quad$ in-situ stress ratio

$\sigma_{\max } \quad$ maximum free-field normal stress

$T_{\max } \quad$ maximum free-field shear stress

$\mathrm{C}_{\mathrm{S}} \quad$ shear wave velocity

$C_{P} \quad$ compressional wave velocity

$\mathrm{n}$ interface friction coefficient

$\delta$ friction angle at the soil-liner interface

e sand void ratio

$\varphi \quad$ sand friction angle 